\title{
A sedação e analgesia de crianças submetidas à ventilação mecânica estariam sendo superestimadas?
}

\author{
Sedation and analgesia in children submitted to mechanical ventilation \\ could be overestimated?
}

\author{
Ana Sfoggia ${ }^{1}$, Patrícia Scolari Fontela ${ }^{2}$, Aline Moraes ${ }^{3}$, Fabrício da Silva ${ }^{3}$, \\ Ricardo Bernardi Sober ${ }^{4}$, Roberta B. Noer ${ }^{5}$, Francisco Bruno ${ }^{6}$, Paulo Einloft ${ }^{7}$, \\ Pedro Celiny Ramos Garcia ${ }^{8}$, Jefferson Pedro Piva ${ }^{9}$
}

\section{Resumo}

Objetivo: descrever o perfil de uso de analgésicos e sedativos em crianças submetidas à ventilação mecânica, internadas em uma UTI pediátrica de referência, em um período de 12 meses, avaliando o tempo de uso dessas drogas, as doses diárias utilizadas e a incidência de síndrome de abstinência.

Métodos: estudo de coorte prospectivo (abril de 2001 a março de 2002), envolvendo crianças em ventilação mecânica (via tubo traqueal) por um período superior a 12 horas, com idade entre 30 dias e 15 anos, que tivessem sucesso no processo de extubação (excluídos os óbitos ou aqueles que necessitassem reintubação). Uma equipe não envolvida com a assistência coletava os dados diariamente, até o $28^{\circ}$ dia de ventilação mecânica (tempo máximo de seguimento para aqueles que eventualmente permanecessem um tempo superior a 28 dias em ventilação artificial), sendo o principal desfecho a dose, infundida às $12 \mathrm{~h}$ da manhã, de morfina, fentanil, quetamina e midazolam (assumindo esta como a dose média naquele dia para cada uma destas drogas). O diagnóstico de síndrome de abstinência foi definido através de pesquisa no prontuário (registro do diagnóstico ou tomada de medidas terapêuticas neste sentido) e por entrevista com o médico assistente de cada paciente, realizada nos dias subseqüentes à extubação. A pesquisa foi aprovada pelo Comitê de Ética e Pesquisa do HSL-PUCRS.

\begin{abstract}
Objective: to describe the pattern of analgesic and sedative infusions in children submitted to mechanical ventilation in a regional pediatric intensive care unit during a 12-month period. To compare the use of these drugs among clinical and surgical patients, as well evaluate the influence of the length of use on the average daily doses and on the incidence of abstinence syndrome.

Methods: this cohort study was performed from April 2001 to March 2002, involving children (1 month old to 15 years old) submitted to the mechanical ventilation with a tracheal tube for a period longer than 12 hours and who were successfully extubated (dead patients and those who required reintubation were excluded from the study). A team of professionals not involved with the patient's assistance performed a daily collection of all data up to the $28^{\text {th }}$ day under mechanical ventilation (maximum length of follow up for those who remain longer under mechanical ventilation). The main outcome was the infusion doses of morphine, fentanyl, ketamine and midazolam administered at $12 \mathrm{AM}$ (considering this dose as the average dose for that day). The diagnosis of abstinence syndrome was based on the chart revision (recorded diagnosis or based on the specific antagonist treatment used) and in an interview with the assistant physician on the following days after the extubation. This study was approved by the Ethics and Scientific Committee of the HSL-PUCRS.
\end{abstract}

\footnotetext{
1. Mestranda do Curso de Pós-graduação em Pediatria da Faculdade de Medicina da PUCRS (FAMED-PUCRS), Pediatra Intensivista do Serviço de Terapia Intensiva e Emergência Pediátrica do Hospital São Lucas da PUCRS (HSL-PUCRS).

2. Mestre em Pediatria pelo Curso de Pós-Graduação em Pediatria - FAMED-PUCRS, Pediatra Intensivista da UTI Pediátrica do Hospital Santo Antônio.

3. Bolsista de Iniciação Científica /FAPERGS - FAMED-PUCRS.

4. Bolsista de Iniciação Científica /PUCRS - FAMED-PUCRS.

5. Bolsista de Iniciação Científica /CNPq - FAMED-PUCRS.

6. Prof. Assistente (Mestre) do Dep. Pediatria - FAMED-PUCRS; Médico do Serviço de Terapia Intensiva e Emergência Pediátrica do HSL-PUCRS.

7. Prof. Adjunto (Mestre) do Dep. Pediatria - FAMED-PUCRS; Médico Chefe Associado do Serv. de Terapia Intensiva e Emergência Pediátrica do HSL-PUCRS.

8. Professor Adjunto Doutor do Dep. Pediatria e do Curso de Pós-Graduação em Pediatria - FAMED-PUCRS; Médico Chefe do Serviço de Terapia Intensiva e Emergência Pediátrica do HSL-PUCRS.

9. Professor Adjunto Doutor do Dep. Pediatria e do Curso de Pós-Graduação em Pediatria das Faculdades de Medicina da PUCRS e da UFRGS; Médico Chefe Associado do Serviço de Terapia Intensiva e Emergência Pediátrica do HSL-PUCRS.
}

Artigo submetido em 06.11.02, aceito em 27.05.03. 
Resultados: dos 127 pacientes elegíveis para este estudo, obtivemos dados de 124 pacientes $(16,0 \pm 29,5$ meses, $58 \%$ meninos; 92 definidos como clínicos e 32 como cirúrgicos). Cada criança utilizou uma média de 1,7 sedativos-analgésicos em infusão por dia (sem diferença entre pacientes clínicos e cirúrgicos). Os opióides (morfina e fentanil) foram as drogas mais utilizadas em ambos grupos (fentanil o preferido entre os clínicos, e a morfina entre os cirúrgicos, $\mathrm{p}<0,001)$. O tempo médio de uso diferiu significativamente $(\mathrm{p}<0,01)$ entre os clínicos e cirúrgicos (6,8 contra 3,9 dias), observando-se também que, a partir de 7 dias de uso, houve um aumento significativo $(\mathrm{p}<0,01)$ nas doses de fentanil e midazolam; assim como uma maior prevalência de abstinência $(42 \%)$ no grupo de pacientes clínicos $(\mathrm{p}<0,01)$.

Conclusões: neste estudo que avaliou a prática diária de uma UTI brasileira de referência, pode-se constatar que a infusão contínua de sedativos e analgésicos em crianças submetidas a ventilação mecânica é utilizada de forma liberal (média de 1,7 drogas por paciente por dia) e, principalmente, que os pacientes clínicos utilizam estas drogas por um período maior, o que pode explicar a elevada prevalência de síndrome de abstinência neste grupo.

J Pediatr (Rio J) 2003;79(4):343-8: ventilação mecânica, sedativos, analgésicos, morfina, UTI pediátrica.
Results: 127 children were eligible for this study, but only 124 patients were analyzed $(16.0 \pm 29-5$ months old; $58 \%$ male; 92 defined as clinical patients and 32 as surgical patients). An average of 1.7 sedative-analgesic infusion per patient a day was used in the whole group (without difference between clinical and surgical groups). Morphine and fentanyl were the most common drugs infused in both groups (fentanyl was preferred for the clinical group and morphine for the surgical group). The mean length of infusion was different $(p<0.01)$ between clinical and surgical patients $(6.8$ and 3.9 days, respectively). After the $7^{\text {th }}$ day, there was a significant increase in the fentanyl and midazolam doses $(\mathrm{p}<0.01)$, as well as a higher incidence of abstinence syndrome in the clinical group $(\mathrm{p}<0.01)$.

Conclusions: this study evaluated the daily practice in a regional PICU, and it demonstrated that analgesic and sedative infusions in children submitted to mechanical ventilation are used according to an uncontrolled pattern (average 1.7 drugs/patient/day) and those classified as clinical patients used these drugs for longer periods, what could explain the higher prevalence of abstinence syndrome in this group.

J Pediatr (Rio J) 2003;79(4):343-8: mechanical ventilation, sedative drug, analgesic drug, morphine, Pediatric Intensive Care Unit.

\section{Introdução}

A sedação e a analgesia tornaram-se, nos últimos anos, prioridades no atendimento de pacientes criticamente doentes. Além do bloqueio da percepção nociceptiva, objetiva-se satisfazer as necessidades ansiolíticas, hipnóticas e amnésticas dos pacientes internados em unidades de terapia intensiva pediátrica $\left(\right.$ UTIP) ${ }^{1}$. Falhas em satisfazer essas necessidades têm efeitos desastrosos, pois dores não tratadas induzem ao catabolismo persistente, ativam o sistema nervoso simpático, alteram a demanda cardiovascular, podendo, ainda, desencadear intensa ansiedade e delírio ${ }^{1,2}$.

As necessidades sedativas de cada paciente mudam constantemente, dependendo da natureza e do curso da doença, assim como da interação com outras terapias. A maioria dos agentes sedativos tem ação ansiolítica, hipnótica e amnéstica variável, e com pobre atividade analgésica. Concentrações plasmáticas similares de um sedativo podem produzir efeitos distintos em diferentes pacientes, em decorrência de fatores ligados à farmacodinâmica e à farmacocinética ${ }^{1-3}$.

Sedativos e analgésicos têm sido administrados, preferentemente, sob a forma de infusão contínua em pacientes criticamente enfermos. Entre os mais comumente utilizados em UTIP, encontram-se os benzodiazepínicos (midazolan), os derivados da morfina (morfina e fentanil), os barbitúricos (tiopental) e a quetamina ${ }^{1-9}$.

De uma forma geral, estes agentes, com o uso contínuo e prolongado, induzem à tolerância, que é definida como a necessidade de doses maiores para obter o mesmo efeito sedativo ou analgésico ${ }^{3-5}$. Da mesma forma, pacientes expostos a terapias sedativas, opióides ou não, podem desenvolver neuroadaptação ou dependência fisiológica. Assim, a suspensão rápida desses agentes pode causar sintomas de abstinência, que incluem dilatação de pupilas, sudorese, lacrimejamento, rinorréia, piloereção, taquicardia, vômitos, diarréia, hipertensão, febre, taquipnéia, agitação psicomotora e ansiedade ${ }^{3-5,10}$.

Até este momento, não há um consenso do melhor esquema de sedativos e analgésicos a ser utilizado nas diversas situações que envolvem pacientes críticos. Estudos que avaliaram o perfil de uso de sedativos e analgésicos em UTIs pediátricas têm demonstrado que esta escolha varia de acordo com o tipo de doente a ser tratado, sua farmacocinética e farmacodinâmica, a experiência prévia, fatores econômicos e, inclusive, práticas e tendências locais, baseadas em critérios subjetivos ou indefinidos ${ }^{1,11-13}$. Obviamente, em razão dessa grande variabilidade, ocorre também uma enorme diversidade na incidência de síndrome de abstinência nos diversos centros.

O presente trabalho foi realizado com o objetivo de descrever o perfil de uso de analgésicos e sedativos em crianças submetidas à ventilação mecânica, internadas em uma UTI pediátrica de referência no sul do Brasil, visando a avaliar a influência do tempo de uso dessas drogas nas doses diárias utilizadas e na ocorrência de síndrome de abstinência. 


\section{Material e métodos}

Todas as crianças admitidas à UTI pediátrica do Hospital São Lucas da PUCRS (HSL-PUCRS), entre 01 de abril de 2001 e 31 de março de 2002, e submetidas à ventilação mecânica (VM) por um período superior a 12 horas, foram incluídas em um estudo de coorte prospectivo. A pesquisa foi submetida e aprovada pelo Comitê de Ética e Pesquisa do HSL-PUCRS.

Os critérios de inclusão foram: (a) idade, entre 30 dias e 15 anos; (b) utilizar a ventilação mecânica por mais de 12 horas; (c) realizar o suporte ventilatório através de tubo endotraqueal (pacientes com traqueostomia ou ventilação não invasiva eram excluídos); (d) ser extubado com sucesso da ventilação mecânica (pacientes que evoluíram ao óbito durante a VM, ou que necessitaram de reintubação por obstrução alta ou falha na extubação não tiveram seus dados considerados no presente estudo).

Uma equipe de quatro bolsistas de iniciação científica, e não envolvidos com a assistência, foi especificamente treinada para realizar a coleta diária e ininterrupta dos dados de todos os pacientes qualificados para o estudo, por um período de 12 meses. Eram avaliados: (1) dados de identificação; (2) os diagnósticos, causa da internação na UTIP e da indicação da ventilação mecânica (definir se por doença cirúrgica ou clínica); (3) dose, infundida às 12 horas da manhã, de morfina, fentanil, quetamina e midazolam (assumindo esta como a dose média para aquele dia de cada uma destas drogas). A dose em infusão contínua era registrada em unidades (mg ou $\mu \mathrm{g}$ ) por quilograma de peso por tempo (minuto ou hora), de acordo com o padrão de referência de cada medicamento; (4) após a extubação ou ao $28^{\circ}$ dia de ventilação mecânica, somente houve interesse na coleta de dados referentes à data de alta da UTIP, data da alta do hospital, condições clínicas na alta e definição da presença ou não de síndrome de abstinência. O diagnóstico de síndrome de abstinência foi definido através de pesquisa no prontuário (registro do diagnóstico ou pela tomada de medidas terapêuticas neste sentido, como, por exemplo, prescrição de methadona, clonidina e/ou benzodiazepínicos no período pós-extubação, etc.) e, também, através de entrevista com o médico assistente de cada paciente, que era sempre realizada nos dias subseqüentes à extubação. Naquelas situações que persistisse a dúvida quanto ao possível diagnóstico de síndrome de abstinência, o caso era discutido com os orientadores da pesquisa (JPP e PCRG) quando era então tomada a decisão final.

Aquele paciente que tivesse sido extubado com sucesso, recebido alta para o domicílio e que porventura necessitasse readmissão na UTIP, requerendo ventilação mecânica e sedação, foi considerado, para efeitos desse estudo, como um novo caso. Por outro lado, aquele paciente que tivesse sido extubado e transferido para outra unidade (mas sem alta do hospital) e que retornasse à UTIP, necessitando de ventilação mecânica, esta nova readmissão não seria considerada neste estudo.
Finalizada a coleta, os dados de cada paciente foram transcritos para uma planilha Excel para Windows (Microsoft Office), especialmente programada para esta pesquisa. Os dados quantitativos foram comparados através do teste $t$ de Student, enquanto que os dados categóricos foram comparados através do teste do qui-quadrado ou por testes de associação (risco relativo), elegendo-se um valor de "p" inferior a 5\% como sendo significativo.

\section{Resultados}

Nos doze meses do estudo, foram internados 303 pacientes na UTIP do HSL-PUCRS. Em 164 pacientes $(54,1 \%)$, houve necessidade de utilizar ventilação mecânica por um período superior a 12 horas. Destes, 37 casos não atendiam aos critérios pré-definidos pela pesquisa (27 por óbito durante a ventilação mecânica, cinco por realizar traqueostomia antes da extubação, 3 por falha na extubação por obstrução respiratória alta, 2 por extubação acidental), restando, então, 127 pacientes. Foram computados os dados de 124 pacientes (97,6\% dos elegíveis), porque três prontuários tinham dados incompletos ou insuficientes $(2,4 \%$ de perda).

A idade média dos 124 pacientes foi de $16,0 \pm 29,5$ meses, com 73 crianças $(58,8 \%)$ pertencentes ao gênero masculino. Em 92 pacientes (74,2\%), a indicação da ventilação mecânica foi determinada por uma condição clínica, enquanto que em 32 pacientes $(25,8 \%)$ foi motivada por uma doença cirúrgica. Entre os pacientes clínicos, o motivo da ventilação foi determinado por disfunção do sistema pulmonar (asma, bronquiolite, pneumonia, etc.) em 67 crianças; distúrbios neurológicos (estado epilético, encefalite, etc.) em 10 casos; sepse ou choque séptico em 8 casos; e nos demais, 7 casos por causas diversas (choque cardiogênico, apnéia, obstrução alta). No grupo de pacientes que necessitaram de ventilação mecânica determinada por causa cirúrgica, predominou o pós-operatório de cirurgia cardíaca (20 casos), seguido pelo pós-operatório de cirurgia geral pediátrica ( 9 casos) e pelo pós-operatório de neurocirurgia (3 casos).

Durante o período que estiveram sob ventilação mecânica, estes 124 pacientes utilizaram uma média de 1,72 drogas sedativas e/ou analgésicas em infusão contínua diária; não havendo diferença significativa $(\mathrm{p}=0,2)$ nas médias de drogas infundidas nos grupos clínico e cirúrgico (1,95 versus 1,28 ; respectivamente). Observou-se, ainda, que $80,4 \%$ dos pacientes clínicos e $78,1 \%$ dos pacientes cirúrgicos utilizaram um opióide em infusão contínua (morfina ou fentanil) durante o período de ventilação mecânica, sendo que a morfina foi significativamente mais utilizada entre os pacientes cirúrgicos, enquanto que o fentanil foi o preferido entre os pacientes clínicos $(\mathrm{p}<0,01)$ (Tabela 1). Chama a atenção que a infusão contínua de midazolan foi utilizada em $90 \%$ dos pacientes clínicos contra apenas 50\% dos pacientes cirúrgicos $(\mathrm{p}<0,01)$. 
Tabela 1 - Perfil do uso de analgésicos e sedativos entre pacientes clínicos e cirúrgicos que necessitaram de ventilação mecânica por mais de doze horas

\begin{tabular}{lccc}
\hline & $\begin{array}{c}\text { Clínicos }(\mathbf{n}=\mathbf{9 2}) \\
\mathbf{n}(\boldsymbol{\%})\end{array}$ & $\begin{array}{c}\text { Cirúrgicos }(\mathbf{n}=\mathbf{3 2}) \\
\mathbf{n}(\boldsymbol{\%})\end{array}$ & $\mathbf{p}$ \\
\hline Midazolan & $84(91,3)$ & $16(50,0)$ & $<0,001$ \\
Fentanil & $70(76,1)$ & $14(43,8)$ & $<0,001$ \\
Morfina & $4(4,3)$ & $11(34,4)$ & 0,01 \\
Quetamina & $21(22,8)$ & 0 & 0,01 \\
\hline
\end{tabular}

O tempo médio de uso das drogas foi significativamente maior $(\mathrm{p}=0,002)$ entre os pacientes clínicos $(6,8 \pm 5,0$ dias $)$ do que nos pacientes cirúrgicos ( $3,9 \pm 3,3$ dias), enquanto as doses médias de sedativos e opióides, utilizadas pelos pacientes clínicos e cirúrgicos, não apresentaram diferença significativa (Tabela 2).

A síndrome de abstinência foi diagnosticada em 46 pacientes dos 124 estudados $(34,1 \%)$, havendo uma grande variabilidade e intensidade na apresentação dos sintomas. Entre os 92 pacientes clínicos, que apresentavam um tempo médio de uso de drogas de $6,8 \pm 5,0$ dias, a síndrome de abstinência foi diagnosticada em 45 casos (49\%) contra apenas 1 caso (3\%) nos 32 pacientes cirúrgicos $(\mathrm{p}<0,0001)$, que utilizavam um tempo médio de $3,9 \pm 3,3$ dias; o que representa um risco relativo de 15,65 (IC95\%:2,25-108,96).

Quando analisamos as doses médias diárias destes analgésicos e sedativos de infusão contínua em grupos estratificados pelo tempo de uso (até 3 dias, de 3 a 7 dias e acima de 7 dias), observamos que as doses médias de midazolan e fentanil, empregadas após uma semana de uso, eram significativamente maiores $(\mathrm{p}<0,01)$ que aquelas utilizadas nos primeiros sete dias de uso (Tabela 3 ).

\section{Discussão}

Neste estudo, que avaliou a prática diária do uso de sedativos e analgésicos em infusão contínua, no período de um ano, em crianças submetidas à ventilação mecânica em uma UTI brasileira de referência, pode-se constatar que: (a)

Tabela 2 - Comparação das doses médias utilizadas nos pacientes clínicos e cirúrgicos submetidos à ventilação mecânica por mais de doze horas

\begin{tabular}{lccc}
\hline & $\begin{array}{c}\text { Pacientes clínicos } \\
\text { Dose média } \pm \mathbf{d p}\end{array}$ & $\begin{array}{c}\text { Pacientes cirúrgicos } \\
\text { Dose média } \pm \mathbf{d p}\end{array}$ & p \\
\hline Midazolan $(\mu \mathrm{g} / \mathrm{kg} / \mathrm{hora})$ & $0,546 \pm 0,3$ & $0,424 \pm 0,3$ & 0,172 \\
Fentanil $(\mu \mathrm{g} / \mathrm{kg} / \mathrm{hora})$ & $5,5 \pm 3,8$ & $4,87 \pm 3,3$ & 0,597 \\
Morfina $(\mu \mathrm{g} / \mathrm{kg} / \mathrm{hora})$ & $22,2 \pm 17,0$ & $12,86 \pm 5,3$ & 0,325 \\
Quetamina $(\mu \mathrm{g} / \mathrm{kg} / \mathrm{min})$ & $33,8 \pm 9,6$ & 0 & - \\
\hline
\end{tabular}

Tabela 3 - Relação entre o tempo de uso dos sedativos e analgésicos e a dose média empregada nos pacientes submetidos à ventilação mecânica

\begin{tabular}{|c|c|c|c|c|}
\hline & $<3 \operatorname{dias}($ a) $(\mathrm{A})$ & 3-7 dias (B) & $>7 \operatorname{dias}(\mathrm{C})$ & $\mathbf{p}$ \\
\hline $\begin{array}{l}\text { Midazolam (n) } \\
\text { Média }+\mathrm{dp} \\
\qquad(\mu \mathrm{g} / \mathrm{kg} / \mathrm{h})\end{array}$ & $\begin{array}{c}(26) \\
0,394 \pm 0,288\end{array}$ & $\begin{array}{c}(40) \\
0,460 \pm 0,266\end{array}$ & $\begin{array}{c}(34) \\
0,707 \pm 0,351\end{array}$ & $\begin{array}{c}\mathrm{AxB}=0,355 \\
\mathbf{A x C}=\mathbf{0 , 0 0 0 4} \\
\mathbf{B x C}=\mathbf{0 , 0 0 1}\end{array}$ \\
\hline $\begin{array}{l}\text { Fentanil (n) } \\
\text { Média }+\mathrm{dp} \\
\qquad(\mu \mathrm{g} / \mathrm{kg} / \mathrm{h})\end{array}$ & $\begin{array}{c}(21) \\
4,00 \pm 2,93\end{array}$ & $\begin{array}{c}(34) \\
4,51 \pm 2,82\end{array}$ & $\begin{array}{c}(29) \\
7,31 \pm 4,25\end{array}$ & $\begin{array}{l}\mathrm{AxB}=0,528 \\
\mathbf{A x C}=\mathbf{0 , 0 0 2} \\
\mathbf{B x C}=\mathbf{0 , 0 0 4}\end{array}$ \\
\hline $\begin{array}{l}\text { Morfina (n) } \\
\text { Média }+\mathrm{dp} \\
\qquad(\mu \mathrm{g} / \mathrm{kg} / \mathrm{h})\end{array}$ & $\begin{array}{c}(8) \\
12,18 \pm 3,96\end{array}$ & $\begin{array}{c}(4) \\
15,50 \pm 7,31\end{array}$ & $\begin{array}{c}(3) \\
23,65 \pm 19,15\end{array}$ & $\begin{array}{l}\mathrm{AxB}=0,466 \\
\mathrm{AxC}=0,409 \\
\mathrm{BxC}=0,544\end{array}$ \\
\hline $\begin{array}{l}\text { Ketamina (n) } \\
\text { Média }+\mathrm{dp} \\
\qquad(\mu \mathrm{g} / \mathrm{kg} / \mathrm{min})\end{array}$ & $\begin{array}{c}\text { (3) } \\
36,31 \pm 15,01\end{array}$ & $\begin{array}{c}(5) \\
37,21 \pm 12,41\end{array}$ & $\begin{array}{c}(12) \\
31,89 \pm 7,19\end{array}$ & $\begin{array}{l}\mathrm{AxB}=0,934 \\
\mathrm{AxC}=0,666 \\
\mathrm{~B} \times \mathrm{C}=0,409\end{array}$ \\
\hline
\end{tabular}


essas drogas são utilizadas de forma bastante liberal (média de 1,7 drogas por paciente por dia); (b) os derivados da morfina são utilizados em $80 \%$ destes pacientes, sendo a morfina o preferido para pacientes cirúrgicos, enquanto que o fentanil é o mais utilizado entre pacientes clínicos; (c) a dose média de sedativos e analgésicos não difere entre os pacientes cirúrgicos e clínicos. Entretanto, os pacientes clínicos utilizam essas drogas por um tempo significativamente maior (6,8 dias versus 3,9 dias) e apresentam uma incidência muito maior de síndrome de abstinência (49\% versus 3\%), o que parece estar mais relacionado ao tempo que à condição clínica; (d) após sete dias de uso, tanto o midazolan quanto o fentanil necessitaram de aumentos significativos em suas doses de infusão.

Na concepção e planejamento deste estudo, decidimos avaliar uma população de pacientes que agudamente necessitassem de ventilação mecânica através do tubo traqueal. Decidimos excluir os pacientes ventilados através de traqueostomia, visto que tais pacientes apresentam doenças crônicas e, via de regra, utilizam baixíssimas quantidades de sedativos, o que poderia acarretar um vício na análise dos dados. Da mesma forma, os pacientes que necessitam de reintubação, por falha de extubação ou por extubação acidental, acabam prolongando o seu período de ventilação $\mathrm{e}$, conseqüentemente, utilizando uma quantidade adicional de sedativos, ocasionando um vício na análise dos dados.

Uma possível crítica ao estudo prende-se ao fato de não haver sido mensurado a administração de outros sedativos, tais como hidrato de cloral, ou doses de ataque de midazolan, diazepan, tionembutal, morfina, entre outros. Provavelmente esta administração adicional de tais medicamentos ocorreu, o que poderia demonstrar uma inadequada sedação. Por outro lado, em nosso serviço (a exemplo de outros centros), à medida que tais medicamentos são utilizados de forma intermitente e freqüente, as doses de manutenção do dia subseqüente são aumentadas. Daí a preocupação de tomar como dose de referência a dose prescrita às 12 horas da manhã, o que facilitou e normatizou a coleta dos dados.

Mesmo considerando-se que as drogas e as doses utilizadas obedecem às recomendações propostas pela literatu$\mathrm{ra}^{4,8,10,14-18}$, não há como extrapolar ou considerar estes achados como representativos da totalidade das UTIs pediátricas em nosso meio. Obviamente, estes resultados refletem a realidade de uma unidade específica, devendo ser analisados dentro deste âmbito. Entretanto, como não há uma prática uniforme na utilização destas medicações, pode-se considerar que os dados apresentados aqui podem refletir a prática de um considerável número de unidades.

Nesta casuística, observa-se que o fentanil foi o opióide de eleição entre os pacientes clínicos $(76 \%)$ e cirúrgicos $(43,8 \%)$, sendo a morfina utilizada em muito menores proporções (4\% e $34 \%$, respectivamente). É sabido que a morfina, quando comparada ao fentanil, apresenta algumas desvantagens, tais como promover maior liberação de histamina (induzir ao broncoespasmo) e uma maior incidência de hipotensão. Por outro lado, o fentanil parece induzir com mais frequiência a abstinência e apresentar um custo significativamente maior ${ }^{1-5,8,10,12-18}$. Nossos achados nos levam, obrigatoriamente, a questionar se a maior opção pelo fentanil foi realmente definida por limitações e contraindicações clínicas ao uso de morfina, ou se apenas expressa uma prática assistencial definida de forma aleatória, ou baseada em razões individuais de cada médico e sem um motivo adequadamente embasado.

Entre os pacientes clínicos e cirúrgicos, não se observou diferença significativa nas doses médias de midazolan, opióides e quetamina administradas. Um dado que chama a atenção é que as doses médias de fentanil e morfina infundidas podem ser consideradas como doses baixas, enquanto que as doses médias de midazolan e quetamina situaram-se no limite superior das doses recomendadas ${ }^{9-13,15-18}$. Em nossa análise, acreditamos que estes resultados expressem apenas uma preferência bastante consistente das opções do serviço, mas que não podem ser analisadas quanto a efetividade e segurança.

Parece haver uma evidente mudança na importância dada à sedação e analgesia nos dias de hoje, se comparado com tempos recentemente passados. Observa-se que seu uso tem sido muito mais liberal, como demonstrado no presente estudo, no qual as crianças submetidas à ventilação mecânica utilizaram, em media, 1,7 sedativos analgésicos por dia. Apesar das evidentes vantagens (prover analgesia e conforto), com o uso destes medicamentos nesta situação, existe um outro lado da moeda: o seu potencial mórbido, pois o uso prolongado e excessivo destes medicamentos pode desencadear alterações cardiovasculares (hipotensão e bradicardia), gastrointestinais (íleo), hepáticas, neurológicas e renais ${ }^{10,15-18}$. Paralelamente, alguns estudos demonstraram que o uso de sedativos em infusão contínua aumenta o tempo de ventilação artificial, o tempo de internação em UTI, e prolonga a internação hospitalar em adultos, quando comparados com pacientes não sedados, ou com os que os receberam sob a forma de bolus ${ }^{13}$.

Está suficientemente demonstrado que, além dessas conseqüências, o uso liberal e prolongado de analgésicos e sedativos pode induzir à tolerância e abstinência ${ }^{2-4,10,14}$. Tolerância refere-se à necessidade de aumentar a dose do medicamento para obter o mesmo efeito clínico (perda da sensibilidade ou esgotamento dos receptores, indução de aumento do metabolismo e depuração dos fármacos), enquanto que abstinência refere-se a um conjunto de manifestações comportamentais, autonômicas e motoras (choro constante, sudorese, agitação, tremores, alucinações, irritabilidade, lacrimejamento, salivação, movimentos mastigatórios, etc.) em resposta à suspensão ou diminuição da infusão. A abstinência tem uma relação direta com a dose cumulativa total recebida e, por outro lado, na maioria das vezes, necessita de intervenção terapêutica para antagonizar essas manifestações ${ }^{2-4,10,14,16-18 .}$

No presente estudo, verificou-se que houve um aumento significativo nas doses diárias médias de opióides e mida- 
zolam a partir de uma semana de uso. O que nos faz supor que o ajuste na dose foi em resposta à tolerância desenvolvida. Esta suposição fica reforçada quando observou-se a elevada taxa de abstinência verificada entre os pacientes clínicos (49\%), os quais utilizaram sedativos em infusão contínua, por um longo período $(6,8 \pm 5,0$ dias).

Frente a estes dados, é lícito que se questione se não estaríamos caindo no extremo oposto: sedando excessivamente essas crianças pacientes submetidas à ventilação mecânica? Consideramos seguro e eficaz nosso sistema de avaliação de sedação e analgesia nesses casos? É consenso que as tabelas vigentes para avaliar sedação e analgesia estão longe do padrão considerado ideal ${ }^{17}$. Como não existe um exame ou marcador fidedigno que reflita o "nível ótimo", esta avaliação acaba sendo baseada em interpretações subjetivas, realizadas pelas equipes médicas ${ }^{4,14,17}$. Portanto, com os instrumentos de que dispomos para monitorizar o uso destas drogas, o possível risco de "hipertratamento" não deve ser descartado.

Um outro fator a ser considerado refere-se à necessidade de que novos fármacos venham se incorporar ao arsenal atualmente disponível, com menos efeitos adversos e, principalmente, que sejam seguros e eficazes para uso prolongado, pois a medicação existente, apesar de eficaz a curto prazo, deixa muito a desejar em termos de efeitos adversos, quando utilizada por longos períodos $2,3,10,16,17$.

Por muito tempo, a morfina e o diazepam foram utilizados nesta situação, não apenas por serem as únicas drogas disponíveis, mas também por seu baixo custo ${ }^{11}$. Entretanto, essa realidade vem-se modificando ${ }^{1,2,11,12}$ : (a) a morfina vem progressivamente sendo substituída pelo fentanil, que é um opióide cem vezes mais potente e com menor liberação de histamina do que a morfina; (b) o diazepan vem sendo substituído pelo midazolan, que é um diazepínico com rápido início de ação, ótima distribuição e com maior capacidade amnéstica. Aliados a esses efeitos benéficos, essas medicações são muito mais onerosas e induzem à rápida tolerância (necessitando rápido ajustes nas doses e, consequientemente, aumento maior ainda nos custos) $)^{1,2,16,17}$. Tanto no presente estudo como em outros relatos da literatura, a opção por estes derivados mais caros é observa$\mathrm{da}^{1-4,11,12,17}$. Novamente, deveríamos questionar se esta mudança, além de aumentar os custo, traz consigo vantagens palpáveis (segurança e eficácia?), ou se estaríamos apenas trocando os efeitos colaterais (hipotensão, liberação de histamina) por mais abstinência e tolerância.

Provavelmente, o perfil do uso de sedativos e analgésicos em nosso grupo pode não se aplicar exatamente à realidade de outras unidades de terapia intensiva pediátrica, no entanto, entendemos ser importante veicular esta prática, para que se possa comparar resultados, paraefeitos e se busquem novas alternativas, com menos paraefeitos e melhores efeitos de conforto e sedação a este grupo de pacientes.

\section{Referências bibliográficas}

1. Martin LD, Bratton SL, Quint P, Mayock DE. Prospective documentation of sedative, analgesic and neuromuscular blocking agent use in infants and children in the intensive care unit: a multicenter perspective. Pediatr Crit Care Med 2001;2:205-10.

2. Tobias JD. Tolerance, withdrawal, and physical dependency after long-term sedation and analgesia of children in the pediatric intensive care unit. Crit Care Med 2000;28:2122-32.

3. Lugo RA, MacLaren R, Cash J, Pribble CG, Vernon DD. Enteral methadone to expedite fentanyl discontinuation and prevent opioid abstinence syndrome in the PICU. Phamacotherapy 2001; 21:1566-73.

4. Young C, Knudsen N, Hilton A, Reves JG. Sedation in the intensive care unit. Crit Care Med 2000;28:854-66.

5. Fonsmark L, Rasmussen YH, Carl P. Occurrence of withdrawal in critically ill sedated children. Crit Care Med 1999;27:196-9.

6. Green SM, Denmark TK, Cline J, Roghair C, Abd Allah S, Rothrock SG. Ketamine sedation for pediatric critical care procedures. Pediatr Emerg Care 2001;17:244-8.

7. Youssef-Ahmed MZ, Silver P, Nimkoff L, Sogy M. Continuous infusion of ketamine in the mechanically ventilated children with refractory bronchospasm. Intensive Care Med 1996;22:972-6.

8. Hawryluck LA, Harvey WRC, Lemieux-Charles L, Singer PA. Consensus guidelines on analgesia and sedation in dying intensive care unit patients. BMC Med Ethics [periódico eletrônico] 2002;3. Disponível: www.biomedcentral.com/1472-6939/3/3. Acessado: 15 de julho de 2003.

9. Nehama J, Pass R, Bechtler-Karsch. Continuous ketamine infusion for the treatment of refractory asthma in a mechanically ventilated infant: case report and review of the pediatric literature. Pediatr Emerg Care 1996;12:294-7.

10. Garcia PCR, Einloft PR, Barbosa SF. Sedação pediátrica na UTI. Revista de Medicina da PUCRS 2000;10:237-42.

11. Mathews AJ. An audit of sedation, analgesia and muscle relaxation in pediatric intensive care in the United Kingdom. Paediatr Anaesth 1993;3:107-15.

12. Murdoch S, Cohen A. Intensive care sedation: a review of current British practice. Intensive Care Med 2002;26:922-8.

13. Kollef MH, Levy NT, Ahrens TS, Schaiff R, Prentice D, Sherman G. The use of continuous IV sedation is associated with prolongation of mechanical ventilation. Chest 1998;114:541-8.

14. Rogers M, Muller H, Garcia P. Dor e analgesia em UTIP. In: Piva J, Carvalho P, Garcia P, editores. Terapia Intensiva em Pediatria. $4^{\text {a }}$ ed. Rio de Janeiro: Medsi; 1997.p.570-89.

15. Fields A, Piva J, Garcia P, Amantéa S. Ventilação mecânica. In: Piva J, Carvalho P, Garcia P, editores. Terapia Intensiva em Pediatria. $4^{a}$ ed. Rio de Janeiro: Medsi; 1997.p.197-230.

16. Polaner DM. Sedation-analgesia in the pediatric intensive care unit. Pediatr Clin North Am 2001;48:695-714.

17. Berde CB, Sethna NF. Analgesics for the treatment of pain in children. N Engl J Med 2002;347:1094-103.

18. Jacobi J, Fraser GL, Coursin DB, Riker RR, Fontaine D, Wittbrodt ET, et al. Clinical practice guidelines for the sustained use of sedatives and analgesics in the critically ill adult. Crit Care Med 2002;30:119-41.

Endereço para correspondência:

Dr. Jefferson Pedro Piva

Rua Vicente da Fontoura, 3008/301

CEP 90640-001 - Porto Alegre, RS 